FSCV in ex vivo slice preparations as well as in anesthetized mice in vivo. DA transients were elicited in the dorsolateral striatum (DLS), dorsomedial striatum (DMS); and nucelus accumbens core (NAcc). In some experiments mice were crossed with DAT-Cre animals and channelrhodopsin 2 (ChR2) was virally expressed in DA neurons to allow optical stimulation of DA transients. RESULTS/ ANTICIPATED RESULTS: As previously reported, SAPAP3 KO mice showed excessive grooming compared to control littermates at the age assessed (4-5 months). DA transients evoked by a single electrical pulse in slices from SAPAP3 KO mice were not significantly different from those observed in slices from control littermates in any of the regions tested including the DLS, DMS and NAcc. However, when four electrical pulses were applied at a frequency of $10 \mathrm{~Hz}$ to mimic DA neuron bursting, the magnitude of DA transients observed in the DMS and NAcc of SAPAP3 mice were greater than those evoked in control littermates.Interestingly, phasic stimulation produced similar DA transients in the DLS of both genotypes suggesting that phasic DA signaling was not globally altered. To confirm this finding we crossed SAPAP3 KO mice with DAT-Cre mice and injected ChR2 containing virus into the midbrain to selectively express ChR2 in DA neurons. Transients were then optically evoked resulting in selective activation of DA neurons. Optical stimulation produced a pronounced enhancement of DA release in SAPAP3 KO mice specifically in the DMS and only following phasic-like stimulation. DISCUSSION/SIGNIFICANCE OF IMPACT: These exciting findings suggest that DA signaling in SAPAP3KO mice is dysregulated in a very precise manner that is sub-region specific as well as dependent on the pattern of stimulation. These results suggest that targeted therapies that can modulate these specific modes of dopaminergic signaling in these distinct striatal subregions could provide improved efficacy in OCD patients that are resistant to SSRI treatment.

3439

\section{Role of the Airway Microbiome in Viral Bronchiolitis Associated Respiratory Failure}

Emily Wasserman ${ }^{1}$, Stefan Worgall, M.D., Ph.D. ${ }^{1}$ and

Anurag Sharma, Ph.D. ${ }^{1}$

${ }^{1}$ Weill Cornell

OBJECTIVES/SPECIFIC AIMS: This study aims to determine if a bronchiolitis specific microbiome exists and how it evolves through disease course. Objective 1. Determine the microbiome profile of the airway in virus induced bronchiolitis-associated respiratory failure. Objective 2. Identify changes in the airway microbiome profile through the course of virus induced bronchiolitis associated respiratory failure, and the relationship between microbiome composition and clinical respiratory status. Objective 3. Determine the impact of rhinovirus infection on the lung and stool microbiome in a murine asthma model. METHODS/STUDY POPULATION: Objectives 1 \&2: We are conducting a single-center prospective case-control study of patients admitted to the Komansky - Weill Cornell Pediatric Critical Care Unit. Infants less than two years of age with a diagnosis of bronchiolitis requiring intubation and mechanical ventilation are enrolled as subjects. Infants less than two years of age intubated and requiring mechanical ventilation without primary lung pathology are enrolled as controls. To evaluate our primary objective, tracheal aspirates will be collected from both subjects and controls on the day of intubation. We will perform 16s RNA sequencing on the tracheal aspirate samples and compare the resulting microbiomes. To evaluate secondary objective, we will collect tracheal aspirates of our study population on a daily basis and map the microbiome in parallel with objective measures of respiratory status including oxygen index and successful extubation. Both subjects and controls are being enrolled as a convenience sample. Objective 3: Mice, heterozygous for the sptlc2 gene (Spltc2 +/-) demonstrate reduced de-novo sphingolipids and increased airway hyperresponsiveness with methacholine challenge. Airway hyperresponsiveness is a cardinal feature of asthma. This airway hyperresponsiveness is exacerbated in the setting of rhinovirus (Figure 1). Using 16s sequencing, we will examine the lung microbiome of Sptlc2 +/- ad Sptlc2 +/+ at 1- and 7-days following rhinovirus infection. RESULTS/ANTICIPATED RESULTS: This clinical study is currently IRB approved and enrollment is ongoing. We have enrolled 12 subjects and 5 controls. Sample analysis will begin following the 2018-2019 respiratory season, with an anticipated cohort of 20 subjects and 20 controls. With regards to the murine studies, we have demonstrated that the lung microbiome of Sptlc2 $+/-$ and Sptlc $2+/+$ mice is similar at baseline (Figure 2) and remains similar following 1-day infection with rhinovirus. We do however, see a distinct change in the microbiome profile of the stool of Sptlc2 +/mice following rhinovirus infection (Figure 3). Lung analysis at day 7 post infection is pending. DISCUSSION/SIGNIFICANCE OF IMPACT: These studies will lay the groundwork for detailing the functional role of the airway microbiome in bronchiolitis, with the objective of developing new modalities for disease treatment and prevention. In addition our murine studies allow us to view the microbiome in the context of sphingolipid deficiency, providing a potential mechanistic link to rhinovirus and ORMDL3 associated asthma.

3582

\section{Scavenger Receptor Expression is Differentially Affected by DNAzyme-Gold Nanoparticle Conjugates}

Cory Sylber ${ }^{1}$, Jessica Petree ${ }^{1}$, Nusaiba Baker ${ }^{1}$, Khalid Salaita ${ }^{1}$ and Cherry Wongtrakool ${ }^{1}$

${ }^{1}$ Emory University

OBJECTIVES/SPECIFIC AIMS: Scavenger receptor (SR) surface proteins are highly conserved motifs and are implicated in the uptake of nanotherapies. Gold nanoparticles functionalized with DNAzymes (DzNP) represent a promising novel nanotherapy for lung diseases such as asthma, particularly because they can be delivered directly to the lung. Our lab has been studying the therapeutic potential of a DzNP targeting GATA-3, a master transcription factor regulating Th2 inflammation. Although nanoparticle uptake through scavenger receptors has been described in macrophages in other models, the role of SRs in DzNP uptake in the lung is poorly understood. We hypothesize that scavenger receptors mediate DzNP uptake in alveolar macrophages. To begin examining this hypothesis, we examined whether DzNP exposure and uptake regulates gene expression of MARCO and MSR1, two class A scavenger receptors. METHODS/STUDY POPULATION: Using a silver stain, we measured dose dependent DzNP uptake in murine alveolar macrophages (MH-S). Using qRT-PCR, we measured gene expression of scavenger receptors MSR1 and MARCO in murine alveolar macrophages (MH-S) and after 24 hour exposure to 2251 DzNP, a DzNP targeting GATA-3, and dextran sulfate sodium (DSS), a known SR-A blocker. RESULTS/ANTICIPATED RESULTS: 2251 DzNP uptake in alveolar macrophages is dose dependent. MARCO gene expression levels significantly increase in murine alveolar macrophages when cultured with increasing concentrations of 2251 DzNP (10 pM-2 nM) or DSS 25-200 ug/ml) for 24 hours. However, MSR1 gene expression 